\title{
Dynamic yield stress enhancement in bidisperse magnetorheological fluids
}

\author{
David Kittipoomwong and Daniel J. Klingenberg ${ }^{\text {a) }}$ \\ Department of Chemical and Biological Engineering, University of Wisconsin, \\ 1415 Engineering Drive, Madison, Wisconsin 53706-1607 \\ John C. Ulicny \\ General Motors R\&D and Planning, 30500 Mound Road, Warren, \\ Michigan 48090-0055
}

(Received 30 June 2005; final revision received 31 August 2005)

\begin{abstract}
Synopsis
Particle-level simulations are employed to investigate the rheological properties of bidisperse magnetorheological fluids. These suspensions are treated as nonlinearly magnetizable, neutrally buoyant, non-Brownian spheres immersed in a nonmagnetizable Newtonian continuous phase. We examine the effects of particle size ratio, composition, and field strength on the dynamic yield stress. The dynamic yield stress of bidisperse suspensions is larger than that of monodisperse suspensions at the same particle volume fraction. The smaller particles cause the larger particles to form more chainlike aggregates than those formed in monodisperse suspensions. (C) 2005 The Society of Rheology. [DOI: 10.1122/1.2085175]
\end{abstract}

\section{INTRODUCTION}

Magnetorheological (MR) suspensions are composed of magnetizable particles dispersed in nonmagnetizable media. These suspensions exhibit fast and reversible magnetic field-induced changes in their properties. Induced magnetostatic particle interactions cause the microstructure to change from a fluidlike state to field-aligned fibrous aggregates. The dramatic field-induced structure evolution gives rise to dramatic changes in rheological properties, commonly referred as the "MR effect." MR suspensions can be characterized as Bingham fluids, with magnetic field-dependent yield stresses as large as $100 \mathrm{kPa}$ [Ginder et al. (1996); Jolly et al. (1999)]. The tunable rheological properties make MR fluids attractive for such applications as semiactive shock absorbers, brakes and clutches, actuators, servo-valves, and precision polishing fluids [Jolly et al. (1999); Carlson and Sproston (2000); Klingenberg (2001)]. MR suspensions are the magnetic analog of electrorheological (ER) suspensions, whose rheological properties are enhanced by the application of an electric field [Parthasarathy and Klingenberg (1996); Conrad (1998); Craig (2003)].

Despite the attractive potential of MR based devices, there are still challenges to their

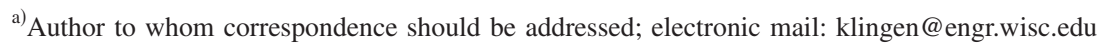


commercialization. One challenge is to obtain the largest possible field-induced yield stress for a minimum energy input in order to reduce the device volume and cost. Increasing the particle concentration is one approach for increasing the yield stress [Lemaire et al. (1995); Genc and Phule (2002)]. However, the off-state viscosity, the suspension viscosity in the absence of an applied field, also increases with volume fraction, which can be problematic in some applications.

Foister (1997) observed that MR suspensions composed of mixtures of magnetizable particles of two different diameters (average diameters 1.25 and $7.9 \mu \mathrm{m}$ ) can exhibit a larger field-induced yield stress than monodisperse suspensions. Such bidisperse suspensions also possess a lower off-state viscosity, which produces a greater change in the shear stress when the field is applied. Similar observations were reported by Weiss et al. (2000) and Ulicny et al. (2003).

Experimental investigations of bidisperse ER suspensions have produced conflicting results. Wu and Conrad (1998) examined the ER response of suspensions of glass spheres of two different sizes (average diameters 6 and $100 \mu \mathrm{m}$ ) in silicone oil, exposed to dc electric fields. Bidisperse suspensions composed of approximately 30-70 vol \% small spheres exhibited smaller yield stresses than monodisperse suspensions composed of either the small or large spheres. The authors postulated that the reduced yield stress may be caused by small particles slipping past the large particles. They also note that at large field strengths, the small particles tended to jump between the electrodes, which suggests that electrophoretic forces may be important. See et al. (2002) observed the opposite trend for bidisperse suspensions of sulfonated poly(styrene-co-divinylbenzene) particles (average diameters 15 and $60 \mu \mathrm{m}$ ) in silicone oil exposed to ac electric fields $(50 \mathrm{~Hz})$. The maximum field-induced shear stresses were obtained for bidisperse mixtures, with the effect being most pronounced at large shear rates. The authors suggest that the enhanced stress transfer may be associated with the increase in packing density that can be achieved with bidisperse suspensions [Cumberland and Crawford (1987)].

The dependence of the MR/ER effect on the particle size distribution may, in some cases, be a result of a direct dependence on particle size; if the response of monodisperse suspensions depends on particle size, then one may observe a dependence on the size distribution. Such a dependence on particle size can arise from a variety of phenomena.

It is well known that Brownian motion can produce a size-dependent MR and ER response. For sufficiently small particles and small field strengths, thermal forces tend to disrupt the field-induced columnar structures. The relative importance of magnetostatic or electrostatic and thermal forces is expressed by the ratios [Adriani and Gast (1988); Lemaire et al. (1995); Baxter-Drayton and Brady (1996)]

$$
\begin{gathered}
\lambda_{B}^{\mathrm{MR}}=\frac{\pi \mu_{0} \mu_{c} a^{3} \beta_{M}^{2} H_{0}^{2}}{2 k T}, \\
\lambda_{B}^{\mathrm{ER}}=\frac{\pi \epsilon_{0} \epsilon_{c} a^{3} \beta^{2} E_{0}^{2}}{2 k T},
\end{gathered}
$$

where $\mu_{0}=4 \pi \times 10^{-7} \mathrm{~N} / \mathrm{A}^{2}$ is the permeability of free space, $\mu_{c}$ is the relative permeability of the continuous phase, $a$ is the particle radius, $\beta_{M}=\left(\mu_{p}-\mu_{c}\right) /\left(\mu_{p}+2 \mu_{c}\right), \mu_{p}$ is the relative permeability of the particles, $H_{0}$ is the applied magnetic field strength, $k$ $=1.381 \times 10^{-23} \mathrm{~J} / \mathrm{K}$ is Boltzmann's constant, $T$ is the absolute temperature, $\epsilon_{0}=8.8542$ $\times 10^{-12} \mathrm{~F} / \mathrm{m}$ is the permittivity of free space, $\epsilon_{c}$ is the relative dielectric constant of the continuous phase, $\beta=\left(\sigma_{p}-\sigma_{c}\right) /\left(\sigma_{p}+2 \sigma_{c}\right)$ (dc fields) or $\left(\epsilon_{p}-\epsilon_{c}\right) /\left(\epsilon_{p}+2 \epsilon_{c}\right)$ (highfrequency ac fields), $\sigma_{p}$ and $\sigma_{c}$ are the electrical conductivities of the particulate and 
continuous phases, $\epsilon_{p}$ is the relative dielectric constant of the particulate phase, and $E_{0}$ is the applied electric field strength. Lemaire et al. (1995) argue that for sufficiently large $\lambda_{B}$, thermal forces should no longer influence the suspension structure, and thus the yield stress should be independent of particle size (independent of $\lambda_{B}$ ). Using their experimental data, they conclude that for $\lambda_{B} \approx 10^{3}$, thermal forces are still strong enough produce a particle size-dependent MR response, but for $\lambda_{B} \approx 10^{9}$, the yield stress is particle size independent. Combining this information with the simulations of Whittle (1990) and Baxter-Drayton and Brady (1996), the "critical" value of $\lambda_{B}$ marking the transition from a particle size-dependent response to a particle size-independent response appears to be between about $4 \times 10^{5}$ and $10^{7}$. In the experimental studies of bidisperse suspensions discussed above, we estimate $\lambda_{B} \geqslant 10^{7}$ for Foister's data, $\lambda_{B} \geqslant 2 \times 10^{5}$ for Wu and Conrad's data, and $\lambda_{B} \geqslant 6 \times 10^{6}$ for See et al.'s data. This suggests that Brownian motion is not playing a role, except perhaps for the smallest field strengths employed (although in each case, shear stresses for the monodisperse suspensions are larger for the large particles than for the small particles).

Other factors can also produce a particle size dependence of the MR and ER responses. Colloidal forces, such as van der Waals attractive and electrostatic repulsive forces, introduce additional scales that can influence the response [Gast and Zukoski (1989)]. In ER suspensions, electrophoresis [Boissy et al. (1995)], surface polarization [Zukoski (1993)], and nonlinear conduction phenomena [Boissy et al. (1996); Felici (1997); Atten et al. (2002)] can also produce particle size dependence.

A few simulation studies have explored the effect of particle size distributions in idealized systems in which the particles experience only field-induced dipolar forces, hydrodynamic forces, and short-range repulsive forces. Wang et al. (1997) simulated shear flow of polydisperse ER suspensions confined to a monolayer. The particle diameter distribution was a truncated Gaussian distribution characterized by the standard deviation. The shear stress decreased monotonically as the standard deviation was increased, for the two dimensionless shear rates examined. The authors attribute the reduced shear stress in polydisperse suspensions to weaker chainlike aggregates. Kittipoomwong et al. (2002) simulated shear flow of bidisperse nonlinearly magnetizable MR suspensions in three dimensions. The yield stress of bidisperse suspensions was larger than that of monodisperse suspensions at the same overall volume fraction.

In this article, we extend the simulation studies reported by Kittipoomwong et al. (2002) to further investigate the role of particle size distribution on the yield stress of bidisperse MR suspensions in which particles interact only via field-induced magnetic forces, hydrodynamic forces, and short-range repulsive forces. The model and simulation method are described in the following section. The yield stress in three-dimensional (3D) systems is greater for bidisperse suspensions than it is for monodisperse suspensions at the same overall volume fraction, in agreement with experimental data reported by Foister (1997). For suspensions confined to a monolayer, the bidisperse suspensions produce a smaller yield stress than that of monodisperse suspensions at the same overall area fraction, consistent with the simulation results of Wang et al. (1997); however, in this case the yield stress scaled by the particle volume fraction within the monolayer is larger for the bidisperse suspensions, consistent with observations for 3D systems. Examination of the microstructure reveals that the enhanced stress transfer in bidisperse suspensions is not associated with an increase in particle packing, but rather with a tendency of the smaller particles to induce the larger particles to form more chainlike aggregates. 


\section{MODEL}

\section{A. Self-consistent mean-field magnetization model}

Application of a magnetic field to a suspension of magnetizable spheres induces magnetic moments on each sphere. The magnetization of each sphere depends on the applied external field as well as the disturbance fields emanating from the neighboring magnetized spheres which must be resolved self-consistently. In this analysis, the magnetization inside each sphere is treated as uniform. As a result, the disturbance field generated by each sphere is treated as a dipolar field. This approximation neglects higher-order multipoles [Klingenberg and Zukoski (1990); Bonnecaze and Brady (1992); Siu et al. (2001)], but approaches the exact solution in the limit of magnetic saturation (i.e., large field strengths) [Davis (1999)].

Consider a collection of magnetizable spheres (bidisperse, diameters $\sigma_{I}, \sigma_{I I}$, relative field-dependent permeability $\mu_{p}$ ), immersed in a nonmagnetizable continuous phase (relative permeability, $\mu_{c}=1$ ), and subjected to a uniform magnetic field $\boldsymbol{H}_{o}=H_{o} \boldsymbol{h}(\boldsymbol{h}$ is a unit vector in the direction of the applied field). The local field experienced by particle $i\left(\boldsymbol{H}_{\mathrm{loc}, i}\right)$ is the applied external field plus the disturbance fields created by all the other spheres,

$$
\boldsymbol{H}_{\mathrm{loc}, i}=\boldsymbol{H}_{o}+\sum_{j \neq i} \boldsymbol{H}_{j}
$$

where $\boldsymbol{H}_{j}$ is the disturbance field created by a particle $j$ at the center of sphere $i$ which can be written

$$
\boldsymbol{H}_{j}=-\mathrm{T}_{i j} \cdot \boldsymbol{m}_{j} .
$$

Here, $T$ is the dipole-dipole interaction tensor [Shkel and Klingenberg (2001)],

$$
\mathrm{T}_{i j}=\frac{1}{4 \pi}\left(\frac{\boldsymbol{\delta}}{r_{i j}^{3}}-3 \frac{\boldsymbol{r}_{i j} \boldsymbol{r}_{i j}}{r_{i j}^{5}}\right),
$$

where $\boldsymbol{r}_{i j} \equiv \boldsymbol{r}_{j}-\boldsymbol{r}_{i}$ is the vector separation between the centers of spheres $i$ and $j$. The magnetic moment induced on sphere $i, \boldsymbol{m}_{i}$, is proportional to the local field $\left(\boldsymbol{H}_{\mathrm{loc}, i}\right)$,

$$
\boldsymbol{m}_{i}=\frac{\pi}{2} \sigma_{i}^{3} \frac{\mu_{p}\left(H_{\mathrm{loc}, i}\right)-1}{\mu_{p}\left(H_{\mathrm{loc}, i}\right)+2}\left[\boldsymbol{H}_{o}-\sum_{j \neq i} \mathrm{~T}_{i j} \cdot \boldsymbol{m}_{j}\right] .
$$

Evaluating the induced moment on each sphere is a formidable task. According to Eq. (6), the induced moment depends on the particle permeability via the relative magnetizability $(\beta)$,

$$
\beta_{i}=\frac{\mu_{p}\left(H_{\mathrm{loc}, i}\right)-1}{\mu_{p}\left(H_{\mathrm{loc}, i}\right)+2}
$$

However, the particle permeability varies nonlinearly with the local field. In this study, the field-dependent permeability is described by the Frohlich-Kennelly constitutive equation [Bozorth (1951)],

$$
\mu_{p}\left(H_{\mathrm{loc}, i}\right)=1+\frac{\left(\mu^{0}-1\right) M_{s} /\left[\left(1-\beta_{i}\right) H_{\mathrm{loc}, i}\right]}{\left(\mu^{0}-1\right)+M_{s} /\left[\left(1-\beta_{i}\right) H_{\mathrm{loc}, i}\right]},
$$

where $M_{s}$ is the saturation magnetization and $\left(1-\beta_{i}\right) H_{\mathrm{loc}, i}$ is the magnetic field strength within sphere $i . \mu^{0}$ is the permeability at zero field, chosen to be $10^{3}$ in this study. 
For a bidisperse system, the moments on spheres of diameter $\sigma_{I}$ and $\sigma_{I I}$ can then be written separately,

$$
\begin{gathered}
\boldsymbol{m}_{\mathrm{I}, i}=\alpha_{\mathrm{I}, i}\left[\boldsymbol{H}_{o}-\sum_{j \neq i}^{I-I} \mathrm{~T}_{i j} \cdot \boldsymbol{m}_{\mathrm{I}, j}-\sum_{j \neq i}^{I-I I} \mathrm{~T}_{i j} \cdot \boldsymbol{m}_{\mathrm{II}, j}\right], \\
\boldsymbol{m}_{\mathrm{II}, i}=\alpha_{\mathrm{II}, i}\left[\boldsymbol{H}_{o}-\sum_{j \neq i}^{I I-I} \mathrm{~T}_{i j} \cdot \boldsymbol{m}_{\mathrm{I}, j}-\sum_{j \neq i}^{I I-I I} \mathrm{~T}_{i j} \cdot \boldsymbol{m}_{\mathrm{II}, j}\right],
\end{gathered}
$$

where $\alpha_{\mathrm{I}, i}=(\pi / 2) \sigma_{I}^{3} \beta_{i}$ and $\alpha_{\mathrm{II}, i}=(\pi / 2) \sigma_{I I}^{3} \beta_{i}$ are the magnetizabilities of sphere $i$ with diameter $\sigma_{I}$ and $\sigma_{I I}$, respectively. The notation $\Sigma_{j \neq i}^{A-B}$ indicates a summation over all neighboring spheres $j$ of type $B$, excluding sphere $i$ of type $A$.

To make the problem more tractable, the following assumptions are made. First, we assume that the moments of all spheres of the same size are equivalent, which reduces the number of unknown moments to two $\left(\boldsymbol{m}_{\mathrm{I}}\right.$ and $\left.\boldsymbol{m}_{\mathrm{II}}\right)$. Each induced moment is approximated to equal to the ensemble average of that particular sphere size. Second, we assume that each moment is parallel to the applied field (i.e. $\boldsymbol{m}_{\mathrm{I}}=m_{l} \boldsymbol{h}$ ).

Dotting Eqs. (9) and (10) with $\boldsymbol{h}$, and ensemble averaging yields the following equations for the moment magnitudes of two different diameter particles,

$$
\begin{gathered}
m_{I}=\alpha_{I}\left[H_{o}-\sum_{j \neq i}^{I-I}\langle\boldsymbol{h} \cdot \mathbf{T} \cdot \boldsymbol{h}\rangle m_{I}-\sum_{j \neq i}^{I-I I}\langle\boldsymbol{h} \cdot \mathbf{T} \cdot \boldsymbol{h}\rangle m_{I I}\right], \\
m_{I I}=\alpha_{I I}\left[H_{O}-\sum_{j \neq i}^{I I-I}\langle\boldsymbol{h} \cdot \mathbf{T} \cdot \boldsymbol{h}\rangle m_{I}-\sum_{j \neq i}^{I I-I I}\langle\boldsymbol{h} \cdot \mathbf{T} \cdot \boldsymbol{h}\rangle m_{I I}\right] .
\end{gathered}
$$

The above two equations can be resolved for the moment magnitudes as

$$
\begin{gathered}
m_{I}=\alpha_{I} \lambda_{I} H_{o}, \\
m_{I I}=\alpha_{I I} \lambda_{I I} H_{o},
\end{gathered}
$$

where the local field enhancement factors $\left(\lambda_{I}\right.$ and $\left.\lambda_{I I}\right)$ are defined

$$
\begin{aligned}
& \lambda_{I}=\frac{1+\alpha_{I I}\left(T_{h}^{I I-I I}-T_{h}^{I-I I}\right)}{1+\alpha_{I} T_{h}^{I-I}+\alpha_{I I} T_{h}^{I I-I I}+\alpha_{I} \alpha_{I I}\left(T_{h}^{I-I} T_{h}^{I-I I}-T_{h}^{I-I I} T_{h}^{I I-I}\right)}, \\
& \lambda_{I I}=\frac{1+\alpha_{I}\left(T_{h}^{I-I}-T_{h}^{I I-I}\right)}{1+\alpha_{I} T_{h}^{I-I}+\alpha_{I I} T_{h}^{I I-I I}+\alpha_{I} \alpha_{I I}\left(T_{h}^{I-I} T_{h}^{I I-I I}-T_{h}^{I-I I} T_{h}^{I I-I}\right)},
\end{aligned}
$$

and the terms $T_{h}^{A-B}$ are defined

$$
T_{h}^{A-B}=\sum_{j(B) \neq i(A)}\langle\boldsymbol{h} \cdot T \cdot \boldsymbol{h}\rangle .
$$

The renormalization procedure proposed by Jeffery (1973) and employed for ER fluids by Adriani and Gast (1988) was employed to resolve the conditionally convergent summations of the term $\langle\boldsymbol{h} \cdot \mathbf{T} \cdot \boldsymbol{h}\rangle$. Using this renormalization procedure to first order in particle volume fraction, the terms $T_{h}^{A-B}$ can be rewritten 


$$
T_{h}^{A-B}=-\frac{\rho^{A}}{3}-\frac{\rho^{A}}{4 \pi} \int^{\infty} \frac{1}{r^{3}}\left(3 \cos ^{2} \theta-1\right)\left(g^{A-B}(\mathbf{r})-1\right) d \mathbf{r},
$$

where $g^{A-B}$ is the pair distribution function for spheres of type $A$ and $B$, and $\rho^{A}$ is the number density of spheres of type $A$.

\section{B. Simulation method}

MR suspensions are modeled as $N$ neutrally buoyant, magnetizable spheres immersed in a nonmagnetizable continuous phase of viscosity $\eta_{c}$. Particles are treated to be homogeneously magnetized but may have different diameters of $\sigma_{\mathrm{I}}$ and $\sigma_{\mathrm{II}}$ with fielddependent relative permeability $\mu_{p}$.

Consider an MR suspension under the constant applied field $\boldsymbol{H}_{o}$. The sphere motion is determined by Newton's equation of motion. Ignoring particle inertia, the force balance for particle $i$ is

$$
\sum_{\text {forces }} \mathbf{F}_{i}\left(\mathbf{r}_{j}\right)=0
$$

where $\mathbf{F}_{i}$ is the net force acting on sphere $i$ which depends on the positions of all the spheres $\left(\mathbf{r}_{j}\right)$. Forces included in the model are magnetostatic interparticle forces $\left(\mathbf{F}_{i j}^{\mathrm{mag}}\right)$, hydrodynamic forces $\left(\mathbf{F}_{i}^{\text {hyd }}\right)$, and short-range repulsive forces $\left(\mathbf{F}_{i j}^{\mathrm{rep}}\right)$. These forces are described in more detail below.

The magnetostatic interparticle forces are treated in the point-dipole limit. Employing the self-consistent mean-field magnetization model described in the previous section, the magnetostatic force on sphere $i$ with diameter $\sigma_{i}$ due to sphere $j$ with diameter $\sigma_{j}$ at position $\left(r_{i j}, \theta_{i j}\right)$ in the point-dipole limit is given by

$$
\boldsymbol{F}_{i j}^{\mathrm{mag}}=F_{o}^{\mathrm{mag}}\left(\frac{r_{\mathrm{min}}}{r_{i j}}\right)^{4}\left[\left(3 \cos ^{2} \theta_{i j}-1\right) \boldsymbol{e}_{r}+\sin 2 \theta_{i j} \boldsymbol{e}_{\theta}\right],
$$

where $r_{\min }=\left(\sigma_{i}+\sigma_{j}\right) / 2$ is the minimum sphere separation, $r_{i j}=\left|\boldsymbol{r}_{i}-\boldsymbol{r}_{j}\right|, \boldsymbol{e}_{r}$ and $\boldsymbol{e}_{\theta}$ are unit vectors in the directions of increasing $r_{i j}$ and $\theta_{i j}$, respectively, and

$$
F_{o}^{\mathrm{mag}}=\frac{3 \pi}{16} \mu_{o} \beta_{i} \beta_{j} \lambda_{i} \lambda_{j} H_{o}^{2}\left(\frac{\sigma_{i}^{3} \sigma_{j}^{3}}{r_{\min }^{4}}\right)
$$

is the magnitude of the magnetostatic force. Here, $\lambda_{i}$ and $\lambda_{j}$ are the local field enhancement factors evaluated self-consistently as described in the previous section. As mentioned in the previous section, this dipole approximation becomes exact at large field strengths (i.e., in the limit of magnetic saturation).

The shearing surfaces are treated as a constant magnetic potential surfaces. Hence, the magnetic interaction between a particle and a surface is equivalent to the interaction of the particle and the dipole images of all other particles reflected about the surface.

The hydrodynamic force on each sphere is treated as Stokes' drag, ignoring hydrodynamic interactions between spheres,

$$
\boldsymbol{F}_{i}^{\mathrm{hyd}}=-3 \pi \eta_{c} \sigma_{i}\left[\frac{d \boldsymbol{r}_{i}}{d t}-\boldsymbol{U}^{\infty}\right],
$$

where $\boldsymbol{U}^{\infty}$ is the ambient fluid velocity. Because we are interested in the behavior of suspensions at vanishing shear rates (see below), we expect that hydrodynamic interactions would have little impact on the results. 
A hard-spherelike interaction force between particles is represented by the short-range repulsive function,

$$
\boldsymbol{F}_{i j}^{\mathrm{rep}}=-F_{o}^{\mathrm{rep}} \exp \left[-\kappa \frac{r_{i j}-r_{\min }}{r_{\min }}\right] \boldsymbol{e}_{r},
$$

where the parameter $\kappa$ characterizes the range of the repulsive force, and $F_{o}^{\mathrm{rep}}$ is the force magnitude. In this article, the force magnitude $F_{o}^{\text {rep }}$ is chosen to be equal to $F_{o}^{\text {mag }}$ and the parameter $\kappa$ is set to 100. Short-range repulsive forces between each sphere $i$ and a surface are modeled with a similar short-range function, $\boldsymbol{F}_{i}^{\text {wall }}=F_{o}^{\text {rep }} \exp \left[-\kappa\left(h_{i}-\sigma_{i} / 2\right) /\right.$ $\left.\sigma_{i}\right] \boldsymbol{e}_{n}$, where $h_{i}=L_{z} / 2-\left|z_{i}\right|, L_{z}$ is the bounding surface separation, and $\boldsymbol{e}_{n}$ is the unit normal vector pointing from the bounding surface into the suspension.

The equation of motion is made dimensionless with the following scales:

$$
L_{s}=\sigma_{L}, \quad F_{s}=\frac{\pi}{48} \mu_{o} \sigma_{L}^{2} M_{s}^{2}, \quad t_{s}=144 \eta_{c} \sigma_{L}^{2} / \mu_{o} M_{s}^{2},
$$

where $\sigma_{L}$ is the diameter of the largest sphere. The force scale is chosen to be the asymptotic value of $F_{o}^{\mathrm{mag}}$ in the limit of magnetic saturation $\left(H_{o} / M_{s} \rightarrow \infty\right)$. The dimensionless equation of motion is

$$
\frac{d \boldsymbol{r}_{i}^{*}}{d t^{*}}=\frac{1}{\sigma_{i}^{*}}\left[\sum_{j \neq i}^{N} \boldsymbol{F}_{i j}^{* \text { rep }}+\boldsymbol{F}_{i}^{*, \text { wall }}+\sum_{j \neq i}^{N^{\prime}} \boldsymbol{F}_{i j}^{*} \text {,mag }\right]+\boldsymbol{u}^{*, \infty},
$$

where the asterisks denote the dimensionless quantities and the prime on the last summation indicates that the summation is over all spheres $j \neq i$, as well as dipole images. Equation (25) represents a set of coupled, nonlinear, first-order differential equations governing the spheres' motion. Spheres within $0.05 \sigma_{i}$ of a bounding surface are considered stuck and assume the lateral ( $x$ or $y$ ) velocity of the bounding surface. The motion of a stuck sphere in the $z$ direction is still governed by Eq. (25), and thus spheres can be pulled away from a bounding surface. Particles in sheared ER suspensions have been observed to stick to bounding surfaces [Klingenberg and Zukoski (1990)], and this feature is required to observe a yield stress in these simulations [Klingenberg et al. (1991)].

Particles are randomly placed in the simulation cell of dimensions $L_{x}^{*} \times L_{y}^{*} \times L_{z}^{*}$. Periodic boundaries are located at $x^{*}= \pm L_{x}^{*} / 2$ and at $y^{*}= \pm L_{y}^{*} / 2$. Shearing surfaces are located at $z^{*}= \pm L_{z}^{*} / 2$. The equation of motion [Eq. (25)] for each sphere is integrated numerically using Euler's method. Interparticle forces are evaluated with a cutoff radius $\left(r_{c}^{*}\right)$ of $L_{z}^{*} / 2$.

The local field enhancement factors $\left(\lambda_{i}\right)$ do not vary significantly from one time step to the next. In order to improve computational speed, we only recalculate the enhancement factors every strain interval of 0.05 . The results are found to be insensitive to this interval.

Rheological properties are evaluated from the particle positions obtained from simulations. The nonhydrodynamic contribution to the shear stress in dimensionless form is

$$
\tau_{x z}^{*}=-\frac{1}{V^{*}} \sum_{i} z_{i}^{*} F_{x i}^{*}
$$

where $F_{x i}^{*}$ is the dimensionless $x$ component of the nonhydrodynamic force on sphere $i, z_{i}^{*}$ is the dimensionless $z$ coordinate of sphere $i$, and $V^{*}$ is the dimensionless simulation cell volume. The dynamic yield stress, the shear stress in the limit of zero shear rate, is calculated by the "relaxation" method [Klingenberg et al. (1991)]. 


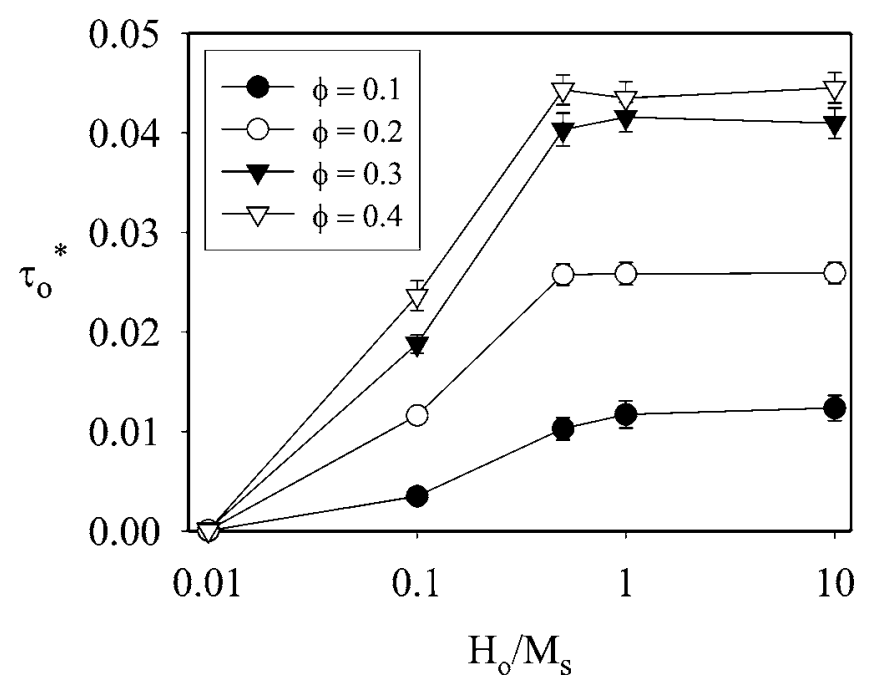

FIG. 1. Dynamic yield stress as a function of normalized field strength for monodisperse suspensions at different particle volume fractions.

Results were averaged over at least five different initial random configurations. Simulations were begun by first integrating the equations of motion with no flow until motion ceased. Shear flow was then simulated at a shear rate of $\dot{\gamma}^{*}=10^{-2}$ to a strain $\gamma=10$. Configurations from these simulations for $\gamma=1-10$ were allowed to relax, and the yield stress was determined from the average of the shear stress of the relaxed configurations. The simulation cell had dimensions $\left(L_{x}^{*}, L_{y}^{*}, L_{z}^{*}\right)=(10,5,5)$ for monodisperse simulations, and dimensions $(5,5,5)$ for bidisperse suspensions; for monolayer simulations $\left(y_{i}=0\right.$ for all $i)$, the simulation cell had dimensions $\left(L_{x}^{*}, L_{z}^{*}\right)=(15,5)$.

\section{RESULTS}

\section{A. Monodisperse suspensions}

The effect of the normalized magnetic field strength on the dynamic yield stress was investigated for particle volume fractions from 0.1 to 0.4 as shown in Fig. 1. The dynamic yield stress increases monotonically with $H_{o} / M_{s}$, and reaches a plateau as the magnetization saturates $\left(H_{o} / M_{s} \geqslant 1\right)$. For $0.01 \leqslant H_{o} / M_{s} \leqslant 1$, the dynamic yield stress from simulations varies subquadratically with field strength. The subquadratic field strength dependence is qualitatively consistent with experimental results reported by Ginder et al. (1996) and Rankin et al. (1999), where the scaling $\tau_{o} \propto H_{o}^{1.5}$ was reported for intermediate field strengths.

According to Fig. 1, the dynamic yield stress becomes independent of field strength at large field strengths $\left(H_{o} / M_{s} \geqslant 1\right)$. The dynamic yield stress at $H_{o} / M_{s}=10$ was selected to represent the dynamic yield stress in the limit of saturated magnetization. The dynamic yield stresses in the limit of saturation are plotted as a function of particle volume fraction in Fig. 2. Dimensional values are obtained by multiplying the scaled yield stresses by $\pi \mu_{o} M_{s}^{2} / 64$. The saturation magnetization value $\mu_{o} M_{s}=2 \mathrm{~T}$, corresponding to that for pure iron [Bozorth (1951)], was employed. The dynamic yield stresses predicted from the simulations are significantly smaller than the static yield stresses obtained from the chain model of Ginder and Davis (1994). Quantitative differences are not unexpected since the simulated structures, while fibrous, are not perfect chains on a lattice. Experi- 


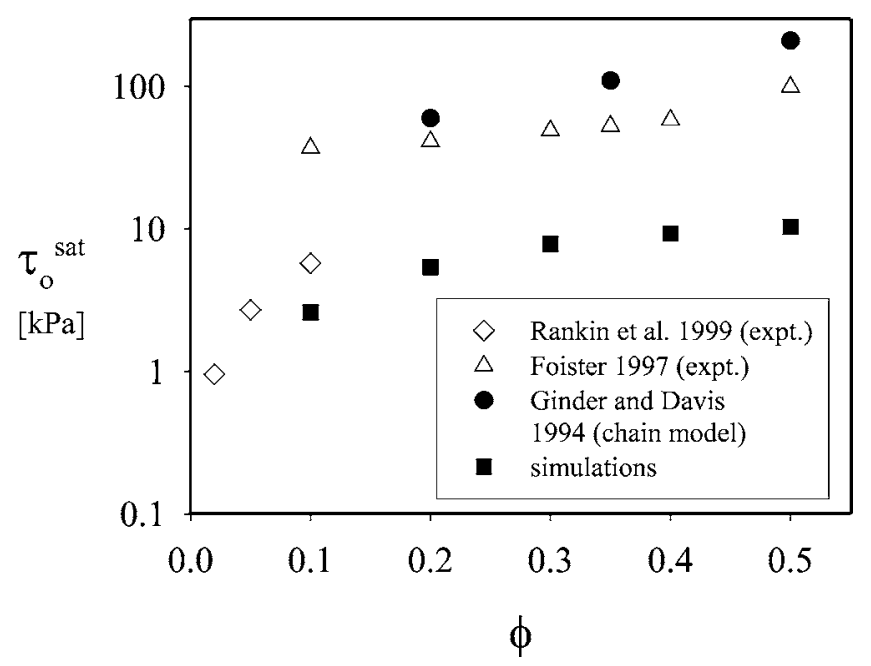

FIG. 2. Yield stress in the limit of magnetic saturation as a function of particle volume fraction for monodisperse suspensions.

mental results by Foister (1997) and Rankin et al. (1999) are included for comparison in Fig. 2. The dynamic yield stress at $\phi=0.1$ is less than the experimentally measured value of approximately $6 \mathrm{kPa}$ reported by Rankin et al. (1999), but is of similar magnitude. Comparing with the data of Foister (1997), our simulation results underestimate the yield stress but exhibit a qualitatively similar volume fraction dependence for low to moderate volume fractions.

\section{B. Bidisperse suspensions}

Simulations in three dimensions were performed for bidisperse suspensions with a particle diameter ratio $\sigma_{L}: \sigma_{S}=2: 1$. The suspension composition is characterized by the fraction of large particles by volume, $\phi_{L} /\left(\phi_{L}+\phi_{S}\right)$, where $\phi_{L}$ and $\phi_{S}$ are the volume fractions of the large and small particle fractions, respectively. Compositions of $\phi_{L} /\left(\phi_{L}+\phi_{S}\right)=0,0.25,0.5,0.75$, and 1 were investigated at various total volume fractions, $\phi=\phi_{L}+\phi_{S}$, and normalized field strengths, $H_{o} / M_{s}$. The dynamic yield stress is plotted as a function of $\phi_{L} / \phi$ in Fig. 3 for simulations at a total volume fraction $\phi=0.4$ and a normalized field strength of $H_{o} / M_{s}=1$. The dynamic yield stress increases as $\phi_{L} / \phi$ is decreased from 1 , reaches a maximum at $\phi_{L} / \phi \approx 0.5$, and then decreases as $\phi_{L} / \phi$ is decreased further. This behavior is consistent with experimental results reported by Foister (1997), who observed larger field-induced yield stresses for bidisperse MR fluids than for monodisperse MR fluids.

The dynamic yield stress is plotted as a function of total volume fraction for simulations of bidisperse suspensions $\left(\sigma_{L}: \sigma_{S}=2: 1\right)$ with $\phi_{L} / \phi=0.75$, as well as for monodisperse suspensions in Fig. $4\left(H_{o} / M_{s}=1\right)$. The dynamic yield stresses of the bidisperse suspensions remain larger than that of the monodisperse suspensions at all concentrations. The dynamic yield stress is plotted as a function of field strength in Fig. 5 for bidisperse $\left(\sigma_{L}: \sigma_{S}=2: 1\right)$ with $\phi_{L} / \phi=0.75$ and monodisperse suspensions for a total volume fraction $\phi=0.4$. Again, we see that the dynamic yield stresses for the bidisperse suspensions remain larger than that of the monodisperse suspensions at all field strengths, even at large field strengths where the magnetization has saturated and the dynamic yield stresses become independent of field strength. 


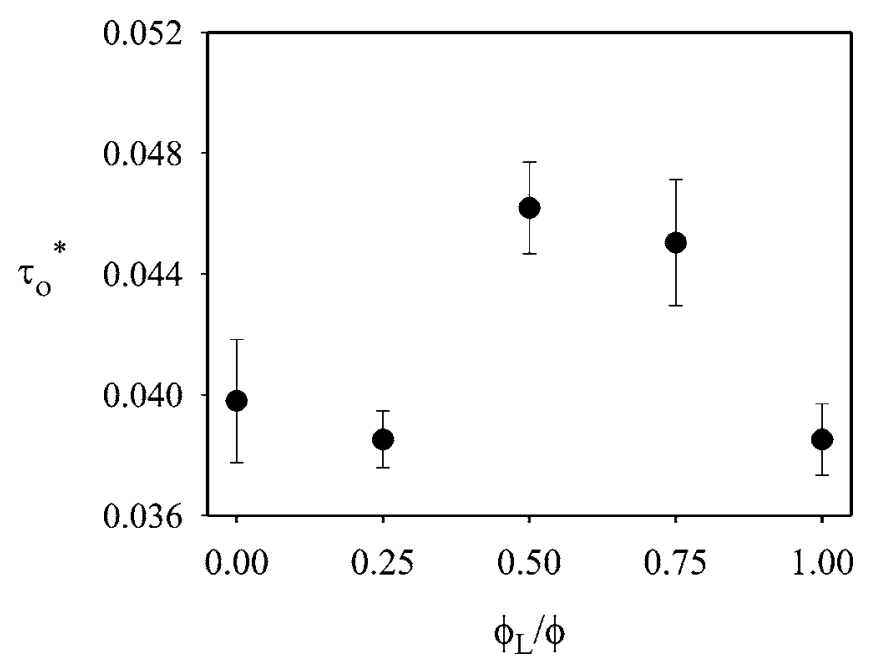

FIG. 3. Dynamic yield stress of bidisperse suspensions as a function of the fraction of large particles by volume $\left(H_{o} / M_{s}=1, \sigma_{L}: \sigma_{S}=2: 1, \phi=0.4\right)$.

\section{DISCUSSION}

The ability to enhance the rheological properties of MR fluids by utilizing polydisperse particle size distributions has obvious implications for improving MR devices. By understanding the mechanisms by which polydispersity achieves this enhancement, one may be able to exploit these mechanisms to further enhance rheological properties.

Equation (26) for the shear stress reveals that polydispersity can influence the shear stress in two ways: By affecting the forces on the particles, and by affecting their relative positions and thus the summation in Eq. (26). Consider first the pair interaction force magnitude,

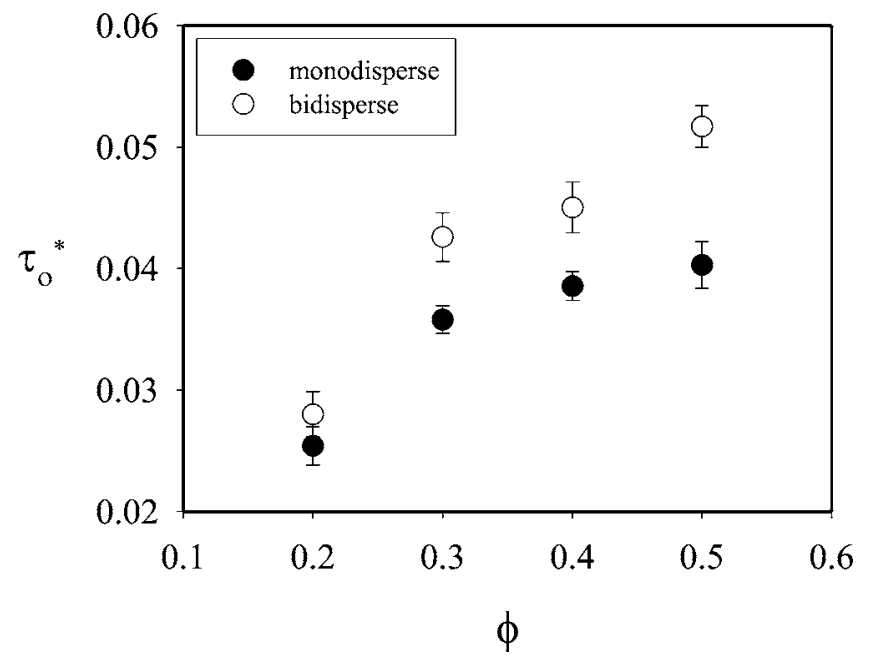

FIG. 4. Dynamic yield stress of monodisperse and bidisperse suspensions as a function of the total particle volume fraction $\left(H_{o} / M_{s}=1, \sigma_{L}: \sigma_{S}=2: 1, \phi_{L} / \phi=0.75\right.$ for bidisperse $)$. 


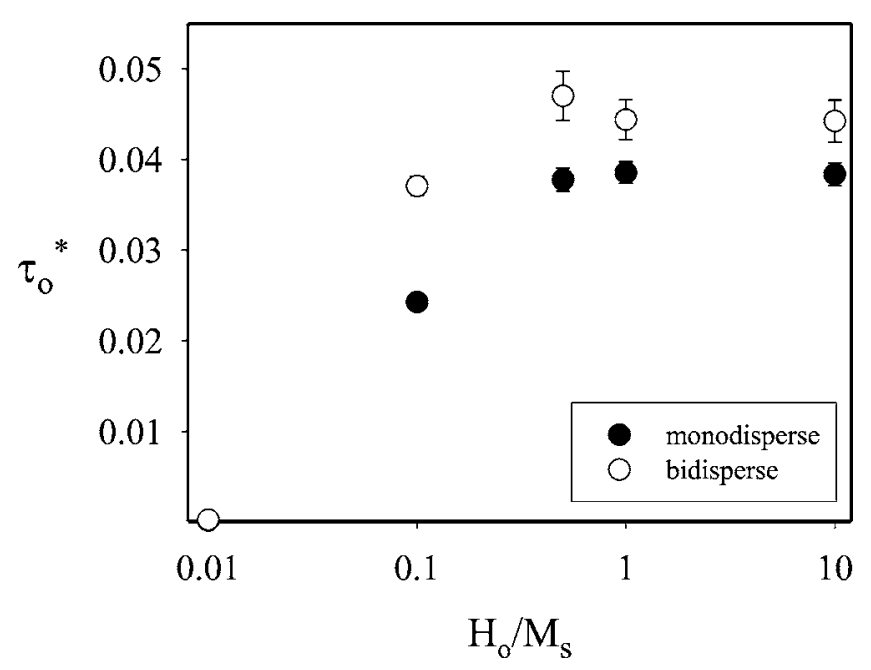

FIG. 5. Dynamic yield stress of monodisperse and bidisperse suspensions as a function of normalized field strength $\left(\sigma_{L}: \sigma_{S}=2: 1, \phi=0.4, \phi_{L} / \phi=0.75\right.$ for bidisperse $)$.

$$
F_{o}^{\mathrm{mag},{ }^{*}}=\frac{F_{o}^{\mathrm{mag}}}{F_{s}}=144 \lambda_{i} \lambda_{j} \beta_{i} \beta_{j}\left(\frac{H_{o}}{M_{s}}\right)^{2}\left(\frac{\sigma_{i}^{* 3} \sigma_{j}^{* 3}}{\left(\sigma_{i}^{*}+\sigma_{j}^{*}\right)^{4}}\right),
$$

where $\sigma_{i}^{*}=\sigma_{i} / \sigma_{L}$. Particle sizes affect this magnitude directly via the last term in parentheses, and indirectly via the effect of particle size distribution on the microstructure and thus the local field, $\lambda_{i}$, and $\beta_{i}$. The last term in parentheses becomes largest for $\sigma_{i}^{*}=\sigma_{j}^{*}$ $=1$, and thus the direct effect of the particle size ratio on the force magnitude can only act to decrease the dynamic yield stress (relative to monodisperse suspensions). Next, consider the indirect effect of particle sizes on the quantity $\lambda_{i} \beta_{i} H_{o} / M_{s}$. In the limit of large field strengths, where the particle magnetization saturates, the quantity $\lambda_{i} \beta_{i} H_{o} / M_{s}$ approaches the constant value $1 / 3$, independent of particle size. In this limit, we still observe an increase in dynamic yield stress for bidisperse suspensions relative to that for monodisperse suspensions at the same total volume fraction. Therefore, the direct and indirect effects of the relative particle sizes on the magnetostatic force magnitude [Eq. (27)] cannot explain the enhancement of the dynamic yield stress for bidisperse suspensions.

The enhancement of the dynamic yield stress for bidisperse suspensions must therefore arise from the changes polydispersity causes in the microstructure. It is well known that the maximum packing fraction of polydisperse suspensions is larger than that of monodisperse suspensions [Cumberland and Crawford (1987)], and thus one might expect that the larger dynamic yield stresses for bidisperse suspension could arise because, locally, the particles may pack more tightly. To probe this, the fluctuations in volume fraction,

$$
\sigma_{\phi}^{2} \equiv\left\langle\phi^{2}\right\rangle-\langle\phi\rangle^{2}
$$

were calculated for the simulated structures. The averages $\left\langle\phi^{2}\right\rangle$ and $\langle\phi\rangle$ were evaluated as follows. The simulation cell was divided into cubes, each of side length $L_{B}$. The volume fraction in each cube was calculated by determining the total volume of particulate material within the cube, and dividing this by $L_{B}^{3}$. The averages $\left\langle\phi^{2}\right\rangle$ and $\langle\phi\rangle$ were equated with the averages of $\phi^{2}$ and $\phi$ for all of the cubes. The fluctuations $\sigma_{\phi}^{2}$ were then 


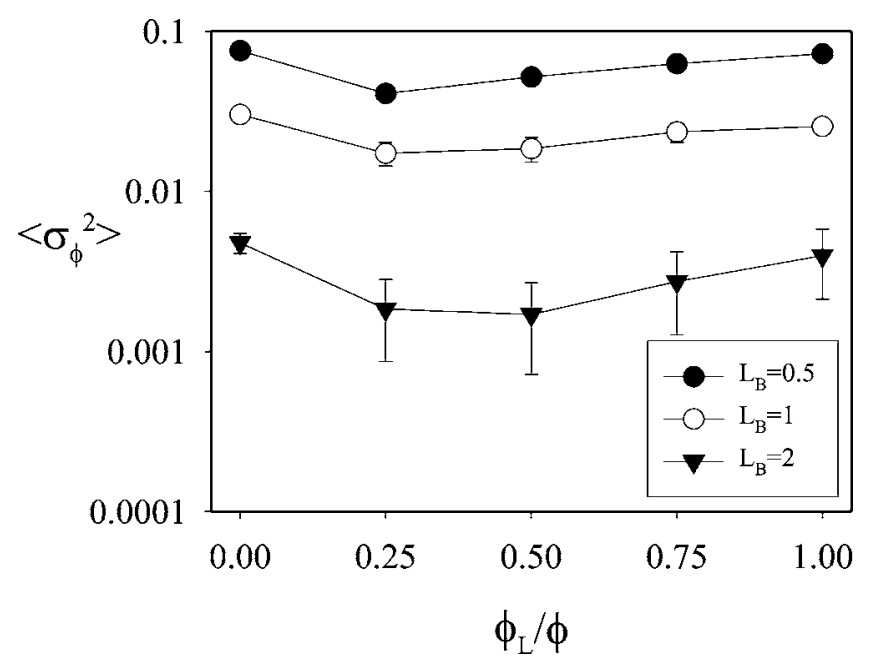

FIG. 6. Volume fraction fluctuations as a function of the fraction of large particles by volume for various sizes of the cubic sampling cell $\left(L_{B}\right)\left(H_{o} / M_{s}=1, \sigma_{L}: \sigma_{S}=2: 1, \phi=0.4\right)$.

averaged over time and different initial configurations. One expects $\sigma_{\phi}^{2}$ to increase as the suspension becomes more heterogeneous. For example, for a well-dispersed system, $\phi$ should be the same in each cube (as long as each cube contains a sufficient number of particles), yielding $\left\langle\phi^{2}\right\rangle=\langle\phi\rangle^{2}$ and $\sigma_{\phi}^{2}=0$. For a heterogeneous system composed of close-packed clusters separated by pure suspending fluid, $\phi$ will vary significantly from one cube to another, yielding $\sigma_{\phi}^{2}>0$.

The volume fraction fluctuations are plotted as a function of $\sigma_{L} / \sigma$ in Fig. 6 for simulations with $\sigma_{L}: \sigma_{S}=2: 1, \sigma_{L}+\sigma_{S}=0.4, H_{o} / M_{s}=1$, and several values of $L_{B}^{*}\left(L_{B}^{*}\right.$ $\left.\equiv L_{B} / \sigma_{L}\right)$. Regardless of the value of $L_{B}^{*}, \sigma_{\phi}^{2}$ is largest for monodisperse suspensions $\left(\phi_{L} / \phi=1\right)$. Thus, the monodisperse suspensions are the most heterogeneous; and while bidisperse suspensions could in principle pack more densely in some regions, they do not in the presence of shear and a magnetic field.

The microstructure of monolayer suspensions is easier to visualize than that of three dimensional systems. In addition, the lower computational cost of monolayer simulations allows simulations to be performed at particle size ratios $\sigma_{L}: \sigma_{S}$ larger than $2: 1$. The dynamic yield stress (left vertical axis) is plotted as a function of particle size ratio $\sigma_{L} / \sigma_{S}$ in Fig. 7 for simulations of monolayer, bidisperse suspensions with $\phi_{L}^{A} / \phi^{A}=0.67$, $H_{o} / M_{S}=1$, and a total particle area fraction $\phi^{A}=\phi_{L}^{A}+\phi_{S}^{A}=0.6$. The dynamic yield stress initially decreases as the particle size ratio $\left(\sigma_{L} / \sigma_{S}\right)$ is increased from 1 (monodisperse) to 2. Although the dynamic yield stress increases for $\sigma_{L} / \sigma_{S}>2$, the dynamic yield stress for $\sigma_{L} / \sigma_{S}=6$ is still slightly smaller than that of the monodisperse suspension. This is consistent with the simulations results reported by Wang et al. (1997) for polydisperse monolayer suspensions.

However, monolayer suspensions at fixed area fraction do not possess the same volume fraction within the monolayer as the particle size ratio is varied, and thus it is not clear how to compare results for monolayer bidisperse suspensions of different particle diameter ratios. Since the yield stress of 3D systems nominally increases with volume fraction (roughly linearly for bidisperse suspensions; Fig. 4), we also plot $\tau_{o}^{*} / \phi$ versus $\sigma_{L} / \sigma_{S}$ in Fig. 7 for the monolayer simulations, where the volume fraction in the monolayer is defined for a simulation cell with thickness $\sigma_{L}$ in the vorticity direction. While 


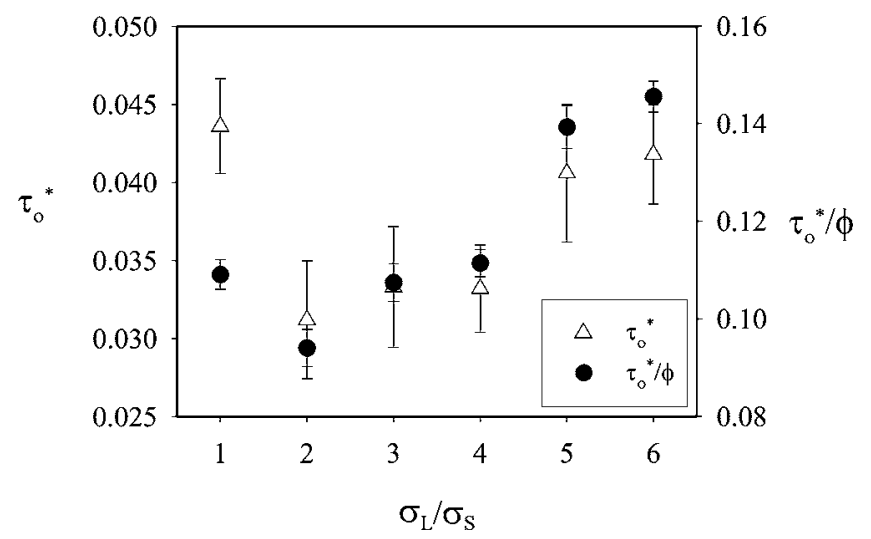

FIG. 7. Dynamic yield stress of monolayer monodisperse and monolayer bidisperse suspensions as a function of particle size ratio $\left(H_{o} / M_{s}=1, \phi^{A}=0.6, \phi_{L}^{A} / \phi^{A}=0.67\right.$ for bidisperse $)$.

$\tau_{o}^{*} / \phi$ initially decreases as $\sigma_{L} / \sigma_{S}$ is increased from 1 to $2, \tau_{o}^{*} / \phi$ then increases, becoming greater than that for monodisperse suspensions for $\sigma_{L} / \sigma_{S} \geqslant 4$. This enhancement is consistent with that observed for 3D systems, and suggests that the mechanisms may be similar.

Snapshots of the simulated microstructure for monolayer suspensions with different particle size ratios are presented in Fig. $8\left(H_{o} / M_{s}=1, \phi^{A}=0.6, \phi_{L}^{A} / \phi^{A}=0.67\right)$. The monodisperse structure appears to consist of large clusters as well as single particle width chains. The bidisperse suspension with $\sigma_{L} / \sigma_{S}=5$ appears to consist largely of single particle width chains of large spheres separated by the smaller spheres. Thus the enhancement of $\tau_{o}^{*} / \phi$ for bidisperse suspensions appears to be associated with a tendency of the larger particles to form more chainlike aggregates.

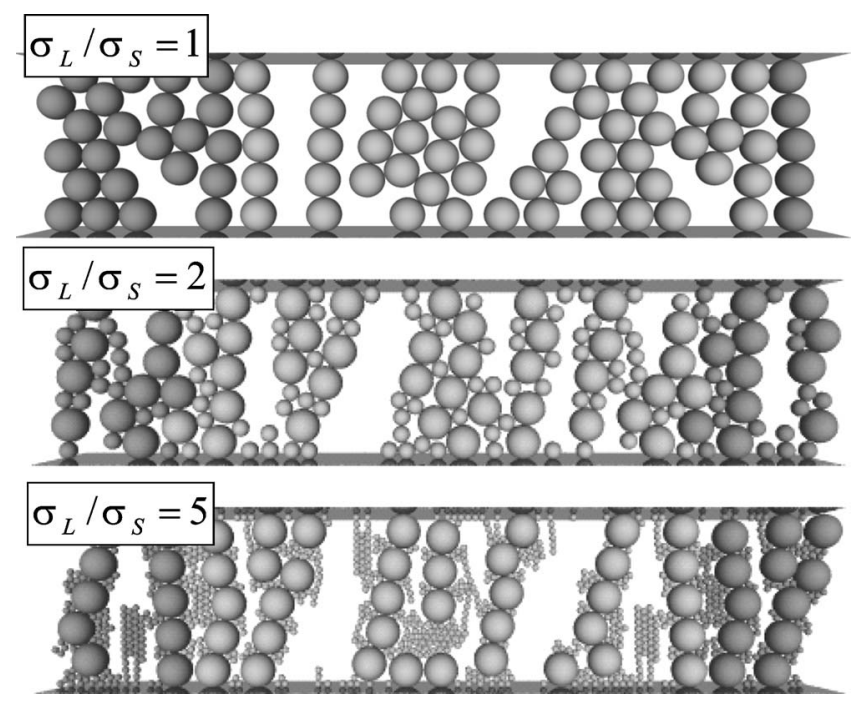

FIG. 8. Snapshots of monolayer monodisperse and monolayer bidisperse suspensions with different particle size ratios (darker shade represents periodic images, $H_{o} / M_{s}=1, \phi^{A}=0.6, \phi_{L}^{A} / \phi^{A}=0.67$ for bidisperse). 
To quantify the degree of structural anisotropy in two- and three-dimensional systems, we analyzed the statistics of the second-order mass moment tensor. The second-order mass moment of the $k$ th cluster composed of $n_{k}$ spheres is defined

$$
\mathbf{I}_{k}=\frac{\sum_{i=1}^{n_{k}} m_{i}\left(\boldsymbol{x}_{i}^{*}-\boldsymbol{x}_{c}^{*, k}\right)\left(\boldsymbol{x}_{i}^{*}-\boldsymbol{x}_{c}^{*, k}\right)}{\sum_{i=1}^{n_{k}} m_{i}} .
$$

Here $\boldsymbol{x}_{i}^{*}$ is the location of the $i^{\text {th }}$ sphere in the cluster, $\boldsymbol{x}_{c}^{*, k}=m_{k}^{-1} \sum_{i=1}^{n_{k}} m_{i} \boldsymbol{x}_{i}^{*}$ is the center of mass of the $k$ th cluster, and $m_{i}=\pi \rho_{m} \sigma_{i}^{3} / 6$ is the mass of the $i$ th sphere. $\rho_{m}$ is the mass density of the particulate material, which is treated as a constant. Clusters were identified using the algorithm described by Sevick et al. (1988), defining two spheres as touching for $\left|\boldsymbol{x}_{i}-\boldsymbol{x}_{j}\right| \leqslant 1.05 r_{\text {min }}$.

The anisotropy of an aggregate can be described in terms of the eigenvalues of the mass moment tensor. Labeling these eigenvalues $I_{1}^{k}, I_{2}^{k}$, and $I_{3}^{k}$ in order of decreasing magnitude ( $I_{1}^{k}$ and $I_{2}^{k}$ for monolayer systems), the degree of anisotropy can be quantified by the ratio

$$
I_{\text {ratio }}^{k}=\frac{I_{1}^{k}}{\sqrt{\left(I_{2}^{k}\right)^{2}+\left(I_{3}^{k}\right)^{2}}}
$$

and $I_{\text {ratio }}^{k}=I_{1}^{k} / I_{2}^{k}$ in monolayer systems. For a perfectly aligned chain, $I_{\text {ratio }} \rightarrow \infty$, whereas for a cubic aggregate of spheres on a cubic lattice, $I_{\text {ratio }}=1 / \sqrt{2}$.

The inverse of the eigenvalue ratios in a given configuration with $N_{c}$ clusters were averaged according to

$$
\left\langle I_{\text {ratio }}^{-1}\right\rangle=\frac{1}{N_{c}} \sum_{1}^{N_{c}}\left(I_{\text {ratio }}^{-1}\right)
$$

so that the presence of a single straight chain with $I_{\text {ratio }} \gg 1$ did not skew the results. These values were then averaged over time and different initial configurations. In evaluating the ratios, we considered clusters consisting of both small and large spheres, as well as clusters of only the large spheres (i.e., by ignoring the small spheres).

The average eigenvalue ratio, $\left\langle I_{\text {ratio }}^{-1}\right\rangle^{-1}$, for monolayer systems is plotted as a function of particle size ratio in Fig. $9\left[H_{o} / M_{S}=1, \phi_{L}^{A}+\phi_{S}^{A}=0.6, \phi_{L}^{A} /\left(\phi_{L}^{A}+\phi_{S}^{A}\right)=0.67\right]$. The average eigenvalue ratios are on the order of one when all spheres are considered when identifying clusters, indicating that these clusters possess a low degree of anisotropy. However, when considering only large spheres in the monolayer suspensions, the average eigenvalue ratio is significantly larger, indicating a high degree of anisotropy as observed in the snapshots of Fig. 8. A similar trend was found for 3D simulations. The average eigenvalue ratio is plotted as a function of $\phi_{L} / \phi$ for $3 \mathrm{D}$ systems in Fig. $10\left(H_{o} / M_{s}\right.$ $\left.=1, \sigma_{L}: \sigma_{S}=2: 1, \phi=0.4\right)$. A much larger value of $\left\langle I_{\text {ratio }}^{-1}\right\rangle^{-1}$ is obtained for clusters of only the large spheres at $\phi_{L} / \phi=0.5$ and 0.75 , compared to that of the monodisperse systems. At these compositions, approximately $70 \%$ of the large spheres are in percolating clusters of large spheres. This is similar to the structure observed for the large diameter spheres in the monolayer system with $\sigma_{L} / \sigma_{S}=5$ depicted in Fig. 8. However, as the small particles become the majority component at $\phi_{L} / \phi=0.25$, there are no longer percolating clusters of large spheres. Increased values of average eigenvalue ratio are also observed for bidisperse suspensions with $\phi_{L} / \phi=0.75, \sigma_{L}: \sigma_{S}=2: 1$ at total volume fractions ranging 


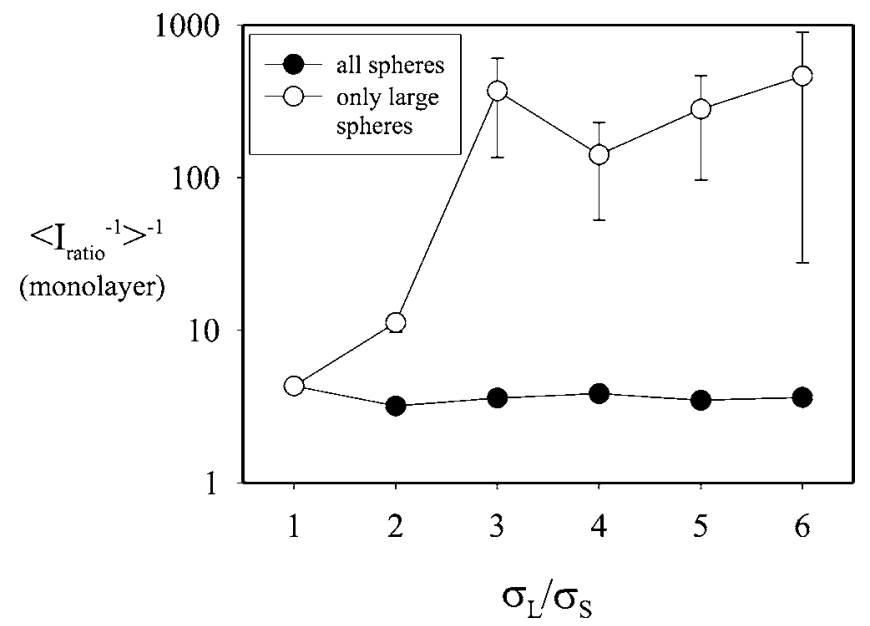

FIG. 9. Average eigenvalue ratio for monolayer systems as a function of particle size ratio for clusters considering all spheres, and for clusters considering only large spheres $\left(H_{o} / M_{s}=1, \phi_{L}^{A}+\phi_{S}^{A}=0.6, \phi_{L}^{A} /\left(\phi_{L}^{A}\right.\right.$ $\left.+\phi_{S}^{A}\right)=0.67$.

from 0.2 to 0.5 . Thus, the enhanced yield stress observed for bidisperse suspensions as depicted in Fig. 4 is associated with the presence of more highly anisotropic clusters of large spheres than observed in monodisperse suspensions.

The tendency of the large particles to form more chainlike aggregates is also reflected in the pair distribution function, $g(r, \theta)$. For a bidisperse suspension, different pair distribution functions can be defined for the distributions of separations of centers of spheres of different types. For example, $g^{L L}(r)$ is the pair distribution function for only the large spheres in a bidisperse system. The pair distribution function of a monodisperse suspension $[g(r, \theta)]$ and the large-large component of the pair distribution function $\left(g^{L L}\right)$ of a bidisperse suspension are presented in Fig. 11. For both suspensions, $\phi=0.2$ and

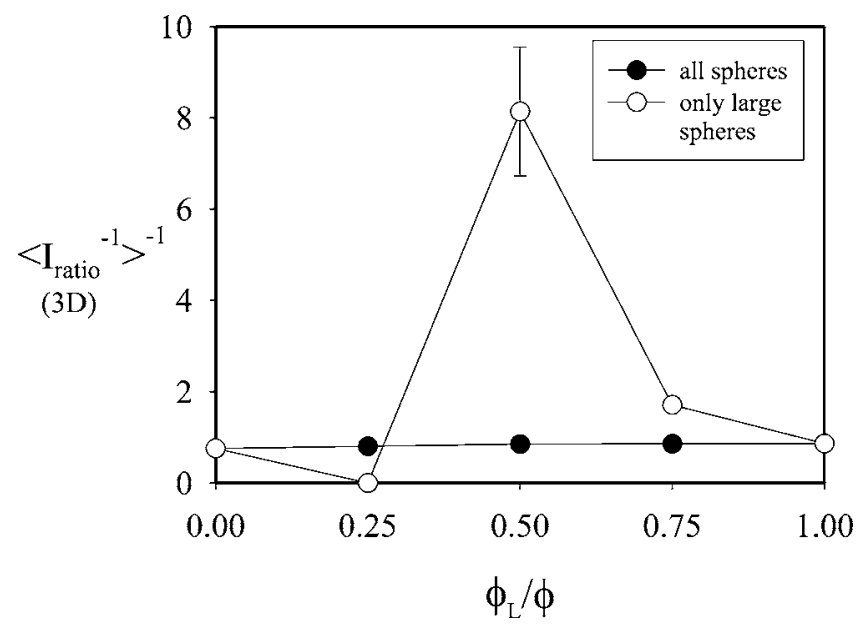

FIG. 10. Average eigenvalue ratio for $3 \mathrm{D}$ systems as a function of the fraction of large particles by volume for clusters considering all spheres, and for clusters considering only large spheres $\left(H_{o} / M_{s}=1, \sigma_{L}: \sigma_{S}=2: 1, \phi\right.$ $=0.4)$. 


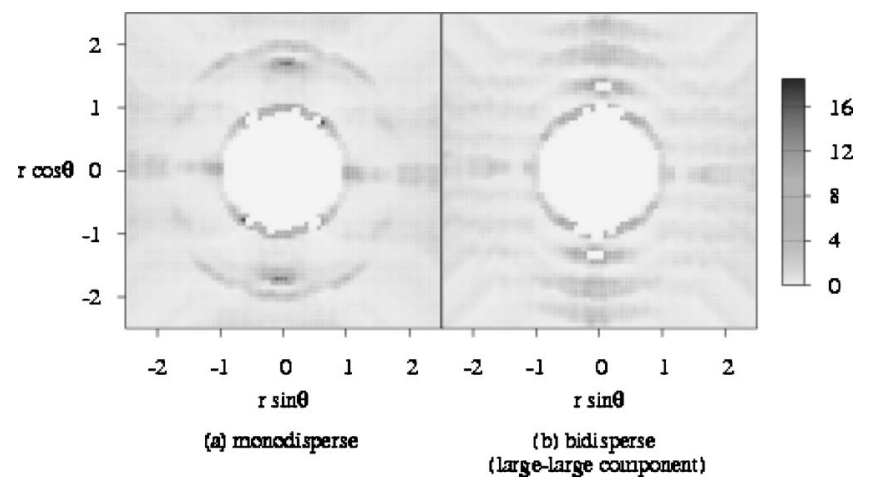

FIG. 11. Pair distribution functions of a monodisperse suspension and a bidisperse suspension (large-large component). For both suspensions, $\phi=0.2$ and $H_{o} / M_{s}=1$. For the bidisperse suspension, $\sigma_{L}: \sigma_{S}=2: 1$ and $\phi_{L} / \phi=0.75$.

$H_{o} / M_{s}=1$. For the bidisperse suspension, $\sigma_{L}: \sigma_{S}=2: 1$ and $\phi_{L} / \phi=0.75$. Both $g$ and $g^{L L}$ exhibit similar patterns of a dense liquid structure with a region of high probability density represented by a dark ring near $r / \sigma_{L}=1$. For the monodisperse suspension, a second ring can be seen at $r / \sigma_{L} \approx 2$. For the bidisperse suspension, $g^{L L}(r, \theta)$ is significantly more anisotropic with a larger probability density near $r \sin \theta=0$. This is consistent with the analysis of the mass moment eigenvalue ratios where bidisperse systems produce more chainlike aggregates of the large spheres.

The observation that higher yield stress in bidisperse MR suspensions, compared to monolayer suspensions of the same volume fraction, are associated with the formation of chainlike aggregates of large spheres does not provide an explanation for why such microstructures produce larger stresses. The stress transfer capabilities of isolated clusters of different shapes (i.e., thin versus thick columns) has been debated [Klingenberg et al. (1991); Kraynik et al. (1992); Gulley and Tao (1993); Anderson (1994)]; the situation here is more complicated because the clusters are not isolated. The mechanisms by which these microstructures produce larger stresses thus remain unknown.

The addition of small particles has also been predicted to influence the microstructure of ferrofluids. In this case, the smaller particles tend to reduce the length of chainlike aggregates of the larger spheres, in contrast to the results reported here in which the aggregate length in the field direction does not change, but the smaller particles tend to inhibit the lateral aggregation of large-sphere chains. Different results, however, are not unexpected since ferrofluids and MR fluids differ in several important respects. Ferrofluid particles are much smaller (diameters $\lesssim 10 \mathrm{~nm}$ ), Brownian, and have permanent magnetic moments. In the modeling studies reported by Kantorovich (2003); Wang and Holm (2003), and Ivanov and Kantorovich (2004), the magnetic interactions between small particles are negligible compared to their thermal energy, and interactions between small and large particles are relatively weak. Aggregate size reduction appears to arise from a poisoning effect, where small particles attach to the ends of the large-particle chains; competition between magnetic interactions and Brownian motion prevent further growth. In MR fluids where Brownian motion is negligible, chain growth is not inhibited, and thus it is not surprising that different effects of the small particles are observed.

\section{CONCLUSION}

We have employed a particle-level simulation technique to probe the effect of particle size distribution on the yield stress of MR suspensions. The particles are modeled as 
neutrally buoyant nonlinearly magnetizable spheres immersed in a Newtonian continuous phase. Simulations of monodisperse suspensions produce yield stresses with a fieldstrength dependence qualitatively consistent with experimental results. Simulations of bidisperse suspensions in three dimensions predict that the yield stress for suspensions of mixtures of small and large spheres is larger than that of monodisperse suspensions at the same volume fraction, in agreement with experimental results reported by Foister (1997).

The microstructure is characterized by the fluctuations in concentration, by the second-order mass moments of clusters within the suspensions, and by the pair distribution functions. We find that the enhanced stress transfer in bidisperse suspensions is not associated with an increase in particle packing, but rather with a tendency of the smaller particles to induce the larger particles to form more chainlike aggregates.

\section{ACKNOWLEDGMENTS}

This work was supported in part by General Motors.

\section{References}

Adriani, P. M., and A. P. Gast, “A microscopic model of electrorheology,” Phys. Fluids 31, 2757-2768 (1988). Anderson, R. A., "Electrostatic forces in an ideal spherical-particle electrorheological fluid," Langmuir 10, 2917-2928 (1994).

Atten, P., J.-N. Foulc, and P. Gonon, "Role and nature of high field conduction of the suspending liquid in electrorheological fluids," in Proceedings of the Eigth International Conference on Electrorheological Fluids and Magnetorheological Suspensions, G. Bossis, ed. World Scientific, Singapore (2002), pp. 752-758.

Baxter-Drayton, Y., and J. F. Brady, "Brownian electrorheological fluids as a model for flocculated dispersions," J. Rheol. 40, 1027-1056 (1996).

Boissy, C., P. Atten, and J.-N. Foulc, "On a negative electrorheological effect," J. Electrost. 35, 13-20 (1995).

Boissy, C., P. Atten, and J.-N. Foulc, "The conduction model of electrorheological effect revisited," Int. J. Mod. Phys. B 10, 2991-3000 (1996).

Bonnecaze, R. T., and J. F. Brady, "Dynamic simulation of an electrorheological fluid," J. Chem. Phys. 96, 2183-2202 (1992).

Bozorth, R., Ferromagnetism (IEEE Press, New York, 1951).

Carlson, J. D., and J. L. Sproston, "Controllable fluids in 2000-status of ER and MR fluids technology," in Actuator 2000-Proceedings of the Seventh International Conference on New Actuators and Drive Systems, edited by H. Borgmann. (Bremen, Germany, 2000), Vols. 19-21, pp. 126-130.

Conrad, H., "Properties and design of electrorheological suspensions," Mater. Res. Bull. 23, 35-42 (1998).

Craig, B., "One fluid, multiple viscosities - numerous applications," AMPTIAC Quart. 7, 15-19 (2003).

Cumberland, D. J., and R. J. Crawford, The Packing of Particles, Handbook of Powder Technology, Vol. 6 (Elsevier, Amsterdam, 1987)

Davis, L. C., "Model of magnetorheological elastomers," J. Appl. Phys. 85, 3348-3351 (1999).

Felici, N. J., "Interfacial effects and electrorheological forces: criticism of the conduction model," J. Electrost. 40,41, 567-572 (1997).

Foister, R. T., "Magnetorheological fluids," U.S. Patent No. 5,667,715 (16 September 1997).

Gast, A. P., and C. F. Zukoski, "Electrorheological fluids as colloidal suspensions," Adv. Colloid Interface Sci. 30, 153-202 (1989).

Genc, S., and P. P. Phule, "Rheological properties of magnetorheological fluids," Smart Mater. Struct. 11, 140-146 (2002).

Ginder, J. M., and L. C. Davis, "Shear stresses in magnetorheological fluids: Role of magnetic saturation," Appl. Phys. Lett. 65, 3410-3412 (1994). 
Ginder, J. M., L. C. Davis, and L. D. Elie, "Rheology of magnetorheological fluids: Models and measurement," Int. J. Mod. Phys. B 23, 3293-3303 (1996).

Gulley, G. L., and R. Tao, "Static shear stress of electrorheological fluids," Phys. Rev. E 48, 2744-2751 (1993).

Ivanov, A. O., and S. S. Kantorovich, "Chain aggregate structure and magnetic birefringence in polydisperse ferrofluids," Phys. Rev. E 70, 021401 (2004).

Jeffery, D. J., "Conduction through a random suspension of spheres," Proc. R. Soc. London, Ser. A A 335, 355-367 (1973).

Jolly, M. R., J. W. Bender, and J. D. Carlson, "Properties and applications of commercial magnetorheological fluids,” J. Intell. Mater. Syst. Struct. 10, 5-13 (1999).

Kantorovich, S. S., "Electronic structure and magnetic properties of gdm ${ }_{2}$ compounds," J. Magn. Magn. Mater. 258,259, 471-473 (2003).

Kittipoomwong, D., D. J. Klingenberg, and J. C. Ulicny, "Simulation of bidisperse magnetorheological fluids," Int. J. Mod. Phys. B 16, 2732-2738 (2002).

Klingenberg, D. J., "Magnetorheology: Applications and challenges,” AIChE J. 47, 246-249 (2001).

Klingenberg, D. J., F. van Swol, and C. F. Zukoski, "The small shear rate response of electrorheological suspensions. I. Simulation in the point-dipole limit,” J. Chem. Phys. 94, 6160-6169 (1991).

Klingenberg, D. J., and C. F. Zukoski, "Studies on the steady-shear behavior of electrorheological suspensions," Langmuir 6, 15-24 (1990).

Kraynik, A. M., R. T. Bonnecaze, and J. F. Brady, "Electrically induced stresses in ER fluids: The role of particle chain structure," in Proceedings of the Third International Conference on ER Fluids, ed. R. Tao (World Scientific, Singapore, 1992), pp. 59-74.

Lemaire, E., A. Meunier, G. Bossis, J. Liu, D. Felt, P. Bashtovoi, and N. Matoussevitch, "Influence of the particle size on the rheology of magnetorheological fluids," J. Rheol. 39, 1011-1020 (1995).

Parthasarathy, M., and D. J. Klingenberg, "Electrorheology: Mechanisms and models," Mater. Sci. Eng., R. 17, 57-103 (1996).

Rankin, P. J., A. T. Horvath, and D. J. Klingenberg, "Magnetorheology in viscoplastic media," Rheol. Acta 38, 471-477 (1999).

See, H., A. Kawai, and F. Ikazaki, "The effect of mixing particles of different size on the electrorheological response under steady shear flow," Rheol. Acta 41, 55-60 (2002).

Sevick, E. M., P. A. Monson, and J. M. Ottino, "Monte Carlo calculations of cluster statistics in continuum models of composite morphology,” J. Chem. Phys. 88, 1198-1206 (1988).

Shkel, Y. M., and D. J. Klingenberg, "Magnetorheology and magnetostriction of isolated chains of ferromagnetic spheres," J. Rheol. 45, 351-368 (2001).

Siu, Y. L., J. T. K. Wan, and K. W. Yu, "Computer simulations of electrorheological fluids in the dipole-induced dipole model," Phys. Rev. E 64, 051506 (2001).

Ulicny, J. C., A. L. Smith, M. A. Golden, B. L. McDermott, and T. J. Chapaton, "Magnetorheological fluids with an additive package," U.S. Patent No. 6,824,701 (30 November 2003).

Wang, Z., and C. Holm, "Structure and magnetic properties of polydisperse ferrofluids: A molecular dynamics study," Phys. Rev. E 68, 041401 (2003).

Wang, Z., Z. Lin, and R. Tao, "Influence of the size distribution of particles on the viscous property of an electrorheological fluid," Chin. Phys. Lett. 14, 151-154 (1997).

Weiss, K. D., J. D. Carlson, and A. D. Nixon, "Method and magnetorheological fluid formulations for increasing the output of a magnetorheological fluid," U.S. Patent No. 6,027,664 (22 February 2000).

Whittle, M., "Computer simulation of an electrorheological fluid,” J. Non-Newtonian Fluid Mech. 37, 233-263 (1990).

Wu, C. W., and H. Conrad, "Influence of mixed particle size on electrorheological response," J. Appl. Phys. 83, 3880-3884 (1998).

Zukoski, C. F., "Material properties and the electrorheological response," Annu. Rev. Mater. Sci. 23, 45-78 (1993). 